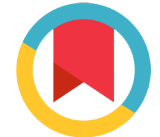

Check for updates
* For correspondence: zahedit1395@gmail.com

Competing interests: The authors declare that no competing interests exist.

Received: 08 January 2018 Accepted: 08 February 2018 Published: 28 February 2018

Copyright The Author(s) 2018. This article is published with open access by BioMedPress.

This article is distributed under the terms of the Creative Commons Attribution License (CC-BY 4.0) which permits any use, distribution, and reproduction in any medium, provided the original author(s) and the source are credited.

\section{Successful treatment with home care during the second half of a twin pregnancy complicated by a short cervix: A case report}

\author{
Mohammad Zarei ${ }^{1}$ (1) Tahereh Zahedifard ${ }^{2, *}$, Reza Nori³ \\ ${ }^{1} \mathrm{MSc}$, Nursing Department, Shirvan Center of Higher Health Education, North \\ Khorasan University of Medical Sciences, Bojnurd, Iran \\ 2MSc, Department of Midwifery, Quchan Branch, Islamic Azad University, Quchan, Iran \\ ${ }^{3} \mathrm{MSc}$, Department of Nursing, Bojnurd Branch, Islamic Azad University, Bojnurd, Iran
}

\begin{abstract}
Introduction: Twin pregnancies are associated with a risk of premature delivery. Case presentation: A 34-year-old primiparous female patient with twin pregnancy presented in the 26th week of pregnancy with a cervical length of $11 \mathrm{~mm}$, with prolapse of membranes. The patient received specialized bed rest and support, and the pregnancy was successfully completed at 36 weeks and 5 days. Conclusion: In the second half of the pregnancy, due to the risk of emergency cervical cerclage, expectant management seems an appropriate and safe approach.
\end{abstract}

\section{Keywords}

Cervical cerclage; Cervix disorders; Home care; Pregnancy

\section{Introduction}

Twin pregnancies have increased significantly in the past decade, mostly due to the use of assisted reproductive techniques. Twin pregnancies are associated with the risk of premature delivery. Almost half of those with a twin pregnancy 
give birth before 37 weeks of pregnancy. The rate is seven times greater than for single pregnancy delivery. The main causes of increased complications of preterm birth and neonatal mortality in twin pregnancies are the long-term morbidity associated with preterm delivery, respiratory distress and infection during the neonatal period (Yazdani et al., 2013).

Incompetent cervix is a risk factor for preterm delivery that can be detected by dilatation of the cervix (with no pain) and prolapse of membranes into the vagina in the second or third trimester of pregnancy. Cerclage is the surgical treatment for cervical insufficiency. Bleeding, uterine contractions, rupture of membranes and cervical dilatation of more than $4 \mathrm{~cm}$ are contraindications for cerclage. When the gestational age is greater, the possibility for surgery to trigger preterm labor is increased. Usually, after 23 weeks of pregnancy, cerclage should be avoided (Cunningham, 2014).

Skupski et al. suggest that cerclage treatment for incompetent cervix in the second half of pregnancy is inferior to bed rest, which allows a significant increase of the gestational age and reduces the risk of preterm birth and its associated complications (Skupski et al., 2014). Although it is believed that there is an association between maternal physical activity and risk of preterm labor, there is no evidence that bed rest is not useful for the patients. The purpose of this report is to present a case of second half twin pregnancy with very short cervical length, which ended successfully with a specialized bed rest at home.

\section{Case Presentation}

A 34-year-old primiparous female was referred to us for the first time after a positive pregnancy test. After the ultrasound at 9 weeks, it became clear that the current twin pregnancy was dizygotic. Due to the high risk of preterm delivery and inadequate cervical length, she was recommended to have an ultrasound examination for cervical length in week 14 of pregnancy. Ultrasonography done at that time reported the pregnancy to be at 14 weeks and the cervical length to be $47 \mathrm{~mm}$. At the next ultrasonography, at 22 weeks of pregnancy, cervical length was $26 \mathrm{~mm}$. The patient was advised to prevent preterm birth and to have cervical cerclage performed; however, she refused the surgical procedure.

At the request of the patient, ultrasonography study of cervical length in the $26^{\text {th }}$ week was conducted, which showed gestational age of 26 weeks and 3 days, and cervical length of $11 \mathrm{~mm}$ with cervical herniation into the amniotic sac. According to the patient's educational status, it was explained that cerclage, at this stage of pregnancy, should not be performed and the patient should just relax at home.

We proposed for the patient administration of heparin to avoid the risks of thromboembolism, yet the patient refused. Therefore, the patient was trained about the effects and risks of thromboembolism. We advised to follow a 
specialized routine which consisted of repeatedly massaging her feet, hands and body, resting in the Trendelenburg position, and moving in the crawl form (allfours) inside the house. She could be in standing position to go to the restroom only once a day. We also trained her to avoid constipation so that less pressure would be exerted on the cervix during a bowel movement.

The patient's weight and blood pressure were checked periodically, and fetal heart rate monitoring and uterine height measurements were done at her home. To avoid depression and boredom and to keep in good mood, the patient was given education about her companion and family in the neonatal period.

Two doses of intramuscular betamethasone were given for assistance of lung maturity, at 24- hour intervals, starting at 28 weeks of pregnancy. With this specialized protocol of home rest, the pregnancy progressed to 36 weeks and 4 days. Finally, the patient returned to the hospital with rapture of the amniotic sac. On examination, cervical dilation was $4 \mathrm{~cm}$ and effacement was $70 \%$ with breech member presentation; thus, the patient was prepared for a caesarean. Two baby boys with Apgar score of 9 were born healthy and without the need for neonatal intensive care. The mother and babies were discharged after a visit by a neonatologist to ensure their health. At follow-up, mother and babies were in good health and reported no problems.

\section{Discusion \& Conclusion}

The cervical length reduction speed of twin pregnancies is greater than that for singleton pregnancy. In our case, there was a higher rate of cervical length, of about $3 \mathrm{~mm}$ per week, which culminated to $11 \mathrm{~mm}$ at the end of the 26th week of pregnancy. If a patient is diagnosed with uterine cervical incompetence and risk of preterm labor, there are two approaches available, namely therapeutic cerclage and bed rest.

Skupski et al., in their 2014 study, concluded that for very short cervix it is better in the second half of pregnancy to choose cervical cerclage over bed rest (Skupski et al., 2014). Crowther et al. (2010) suggested that there is no sufficient evidence to claim that bed rest, for prevention of preterm birth in twins, is effective; however, although bed rest does not reduce mortality and perinatal mortality, it can improve fetal growth (Crowther and Han, 2010). In other studies, Galanaud et al. (2015) reported that bed rest has long been associated with increased risk for thromboembolism (Drife, 2015). However, in our case report patient, since she was advised to perform daily massages in sequence (legs, hands, and body massage), her risk was reduced. Also, considering that the patient was without uterine contractions, we advised her to rest at home and move on all-fours position. This position is better than standing since according to the force of gravity, the weight of the uterus and its contents do not enter the cervix and the patient can move freely in the home. Therefore, several of the 
complications of bed rest, such as thromboembolism, fatigue and muscular atrophy, should be reduced (Maloni, 2010). This position can be used for patients who do not have contractions and only have an incompetent cervix.

Geoffrey et al. (1997) suggest that standing positions, in women with insufficient cervix, cause dilatation of the cervix and prolapse of membranes further into the cervical canal; however, for women with normal cervix the standing position has no effect (Wong et al., 1997). In their study, the patient was advised to rest on the ground, not on the bed, because the patient did not have to stand to move from the bed and, therefore, less pressure would be exerted on the cervix. Saccone et al. (2015) suggested that cerclage should not be performed very frequently in twin pregnancies with short cervical length in the second half of pregnancy, and that any recommendation to do so would require more clinical trials (Saccone et al., 2015).

Despite numerous studies on the management of cervical disease, there is still disagreement about the best treatment, based on the patient's condition and physician's order. However, for advanced stages of pregnancy and older age, and according to emergency cerclage risks (including increased risk of infection and loss of pregnancy), it seems that expectant treatment which includes bed rest is favorable. Keeping a Trendelenburg position at rest, moving around on all-fours, and massage of foot and body can help reduce the risk of thromboembolism. Moreover, administration of corticosteroids for fetal lung maturity and antibiotics, if required, also represents an appropriate and safe solution.

\section{Acknowledgements}

We hereby express our gratitude to Dr. Naebi and the medical staff of Razavi Hospital, Mashhad, Iran, particularly Ms. Ghavamiand Refahi.

\section{Author Contribution}

Zarei and Zahedifard made substantial contributions to the concept and design of the study report, acquisition of data, analysis, and/or interpretation of data. In addition, Zarei and Nori were involved in drafting the manuscript and critically revising it for intellectual content. All authors provided their final approval of the version to be published, and all agreed to be accountable for all aspects of the work, ensuring that questions related to the accuracy or integrity of any part of the work are appropriately investigated and resolved. All authors read and approved the final manuscripts for publishing. 


\section{References}

Crowther, C. A., \& Han, S. (2010). Hospitalisation and bed rest for multiple pregnancy. Cochrane Database of Systematic Reviews, 7(7), CD000110. PMID:20614420

Cunningham, Kenneth Leveno, Steven Bloom (2014) Williams Obstetrics. 24th ed. Newyork: Mc Graw Hill co.

Drife, J. (2015). Deep venous thrombosis and pulmonary embolism in obese women. Best Practice \& Research. Clinical Obstetrics \& Gynaecology, 29(3), 365-376. https:// doi.org/10.1016/j.bpobgyn.2014.08.012 PMID:25457857

Maloni, J. A. (2010). Antepartum bed rest for pregnancy complications: Efficacy and safety for preventing preterm birth. Biological Research for Nursing, 12(2), 106-124. https://doi.org/10.1177/1099800410375978 PMID:20798159

Saccone, G., Rust, O., Althuisius, S., Roman, A., \& Berghella, V. (2015). Cerclage for short cervix in twin pregnancies: Systematic review and meta-analysis of randomized trials using individual patient-level data. Acta Obstetricia et Gynecologica Scandinavica, 94(4), 352-358. https://doi.org/10.1111/aogs.12600 PMID:25644964

Skupski, D. W., Lin, S. N., Reiss, J., \& Eglinton, G. S. (2014). Extremely short cervix in the second trimester: Bed rest or modified Shirodkar cerclage? Journal of Perinatal Medicine, 42(1), 55-59. https://doi.org/10.1515/jpm-2013-0092 PMID:23924522

Wong, G., Levine, D., \& Ludmir, J. (1997). Maternal postural challenge as a functional test for cervical incompetence. Journal of Ultrasound in Medicine, 16(3), 169-175. https://doi.org/10.7863/jum.1997.16.3.169 PMID:9166812

Yazdani, S. H., Zynalzade, M., Bozari, Z., Habibi, S., \& Moradi, M. (2013). The effect of cervical cerclage on prevention of preterm labor in twin pregnancy. [Persian.]. Majallahi Zanan, Mamai va Nazai-i Iran, 16(62), 6-10. 